\title{
An Interstitial 17q11.2 de novo Deletion Involving the CDK5R1 Gene in a High-Functioning Autistic Patient
}

\author{
Carla Lintas $^{a, b} \quad$ Roberto Sacco $^{a, b} \quad$ Claudio Tabolacci $^{b} \quad$ Claudia Brogna $^{a}$ \\ Marco Canali ${ }^{b}$ Chiara Picinellic Pasquale Tomaiuolo ${ }^{c}$ Paola Castronovo ${ }^{c}$ \\ Marco Baccarinc Antonio M. Persicoc, d \\ a Service for Neurodevelopmental Disorders and ${ }^{\mathrm{b}}$ Laboratory of Molecular Psychiatry and Neurogenetics, \\ Department of Medicine, University Campus Bio-Medico, Rome, 'Mafalda Luce Center for Pervasive Developmental \\ Disorders, Milan, and dinterdepartmental Program "Autism 0-90," "G. Martino" University Hospital, University of \\ Messina, Messina, Italy
}

\section{Established Facts}

- The CDK5R1 gene has a pivotal role in neurodevelopment and postnatally in spatial learning and longterm plasticity.

- CDK5R1 has been proposed as a candidate gene for intellectual disability in the NF1 microdeletion syndrome and in idiopathic mental retardation.

- However, in vitro functional studies on CDK5R1 gene variants have shown controversial results relative to a possible role of CDK5R1 in intellectual disability.

\section{Novel Insights}

- This is the first report that associates a CDK5R1 heterozygous deletion with autism spectrum disorder.

- The reported genotype-phenotype association in our patient is strongly supported by the CreER-p35 conditional knockout mouse model and by expression data.

- Our data show for the first time that loss of one copy of the CDK5R1 gene is not correlated with intellectual disability.

\section{Keywords}

CDK5R1 $\cdot$ Haploinsufficiency $\cdot$ High-functioning autism • Intellectual disability

\section{Abstract}

We describe a 32-year-old male patient diagnosed with high-functioning autism spectrum disorder carrying a de novo 196-kb interstitial deletion at chromosome 17q11.2. The deletion was detected by array CGH (180K Agilent) and

\section{KARGER}

(C) 2018 S. Karger AG, Basel

E-Mail karger@karger.com

www.karger.com/msy
Carla Lintas

Laboratory of Molecular Psychiatry and Neurogenetics Department of Medicine, University Campus Bio-Medico

Via Alvaro del Portillo 21, IT-00128 Rome (Italy)

E-Mail c.lintas@unicampus.it 
confirmed by quantitative PCR on genomic DNA. The deleted region spans the entire PSMD11 and CDK5R1 genes and partially the MYO1D gene. The CDK5R1 gene encodes for a regulatory subunit of the cyclin-dependent kinase 5 responsible for its brain-specific activation. This gene has been previously associated with intellectual disability in humans. A reduction in CDK5R1 transcript was detected, consistent with the genomic deletion. Based on the functional role of $C D K 5 R 1$, this gene appears as the best candidate to explain the clinical phenotype of our patient, whose neuropsychological profile has more resemblance with some of the higher brain function anomalies recently described in the CreERp35 conditional knockout mouse model than previously described patients with intellectual disability.

(c) 2018 S. Karger AG, Basel

Autism spectrum disorder (ASD) is a genetically heterogeneous neurodevelopmental condition characterized by repetitive and stereotyped behaviors, restricted interests, and social and communication deficits [American Psychiatric Association, 2013]. Its clinical phenotype is extremely variable, ranging from lack of expressive language in comorbidity with severe intellectual disability, to social deficits and narrowed interests in high-functioning individuals with fluent expressive language and normal IQ. The majority of ASD forms are currently considered a multifactorial disorder with a strong genetic component, in which rare and common variants with variable levels of penetrance interact among themselves and with environmental factors [Persico and Napolioni, 2013]. New genome-wide technologies, such as CGH and SNP arrays as well as NGS, have allowed the identification of many candidate ASD genes in large cohorts. The Simons Foundation website (https://www.simonsfoundation.org), one of the most exhaustive publicly available web resources concerning autism genetic research, currently lists 813 autism candidate genes ranked into 7 main categories based on different levels of evidence, supporting the role of each gene in autism pathogenesis. We hereby report on a young man diagnosed with high-functioning ASD due to social skill deficits and anxiety, who underwent a thorough medical assessment including genetic testing.

\section{Clinical Report}

A 32-year-old man was referred to our department due to social skills deficit and an anxiety disorder. He is the first child of healthy unrelated parents and was born at 42 weeks of gestation by normal
Table 1. Neuropsychological test results

\begin{tabular}{ll}
\hline Cognitive test & $\begin{array}{l}\text { Score or level of } \\
\text { performance }\end{array}$ \\
\hline WAIS-IV & \\
$\quad$ Verbal IQ & 94 (normal) \\
Visuo-perceptive reasoning IQ & 121 (normal) \\
Working memory & 77 (poor) \\
Visuo-motor speed & 86 (normal) \\
Full scale IQ & 95 (normal) \\
Executive functions & \\
Trail making test & Normal \\
Wisconsin card sorting test/set-shifting ability & Normal \\
Tower of London & Normal \\
Elithorn perceptual maze test & Normal \\
Phonological fluency & Poor \\
Memory & \\
Short-term verbal memory & Poor \\
Verbal episodic memory & Normal \\
Verbal learning & Normal \\
Visuo-spatial memory & Normal \\
Attention & \\
Sustained visual attention & Normal \\
Visuo-constructional abilities & \\
Rey complex figure & Normal \\
Arithmetical abilities & \\
Mental calculus & Normal \\
Mathematical reasoning & Normal \\
ADOS-2 & \\
Language and communication & 3 \\
Social interaction & 9 \\
\hline ADOS-2 Aint & \\
\hline
\end{tabular}

ADOS-2, Autism Diagnostic Observation Schedule; WAIS, Wechsler Adult Intelligence Scale.

delivery. Birthweight was 4,200 g. He was exclusively breastfed for 3 months; weaning was accomplished around the fourth month without major problems. Developmental milestones and physical growth were achieved normally. Babbling started at 6 months. Expressive language for single words started at 14 months and first sentences at 20 months. At about 18 months, psychomotor clumsiness was noted for the first time. The child fell down frequently while running; ocular motor deficits, balance problems, and difficulties going up and down the stairs were also reported. Behavioral disturbances including a tendency to isolate from his peers, poor communication, and separation anxiety from his mother were first noted when he started attending kindergarten. As a child, he would tend to obsessively play the same games over and over again. Pain hyposensitivity was also reported. During elementary school, separation anxiety was still present. Difficulties in homework performance, comprehension, and visual-motor coordination in handwriting as well as attention deficit and oppositional traits were observed by parents and teachers. Repetitive and stereotyped patterns of behavior (body and leg sway, toe running, opening and closing his fists), restricted interests and activities were first noted when he was a child and persisted into adulthood. He also displayed emotional self-regulation deficits with psychomotor agitation when frustrated. As an adult, he has achieved a satisfactory level of personal autonomy: he lives with his parents and works as a warehouseman. His EEG was normal, and no dys- 
A
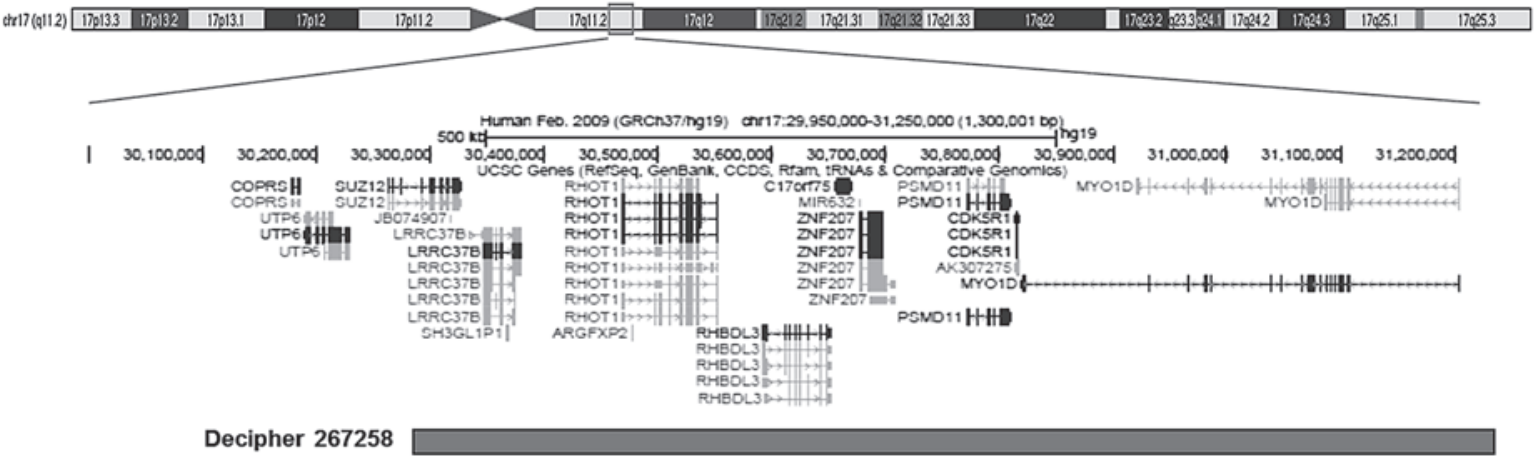

Decipher 289944

Decipher 317985

B

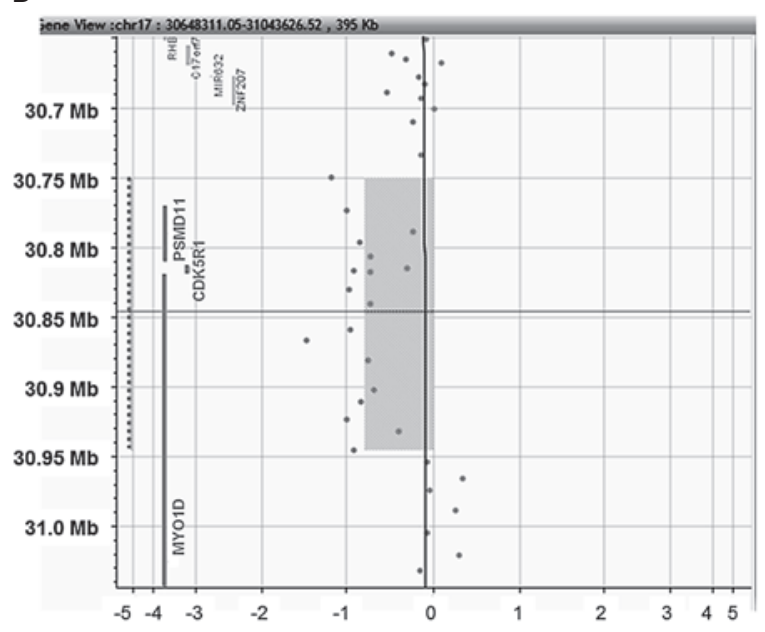

Fig. 1. A Chromosomal location of the 17q11.2 deletion and comparison between the CNV detected in our proband (PB) and 3 CNVs reported in the DECIPHER database [Osio et al., 2018]. Our observed deletion (light gray bar) partially overlaps with the others (dark gray bars) involving the PSMD11, CDK5R1, and MYO1D genes only. The figure is modified from UCSC genome browser (https://genome.ucsc.edu/). B Array-CGH profile of our patient at chromosome band 17q11.2 between 30,750,126 and 30,946,150 bp

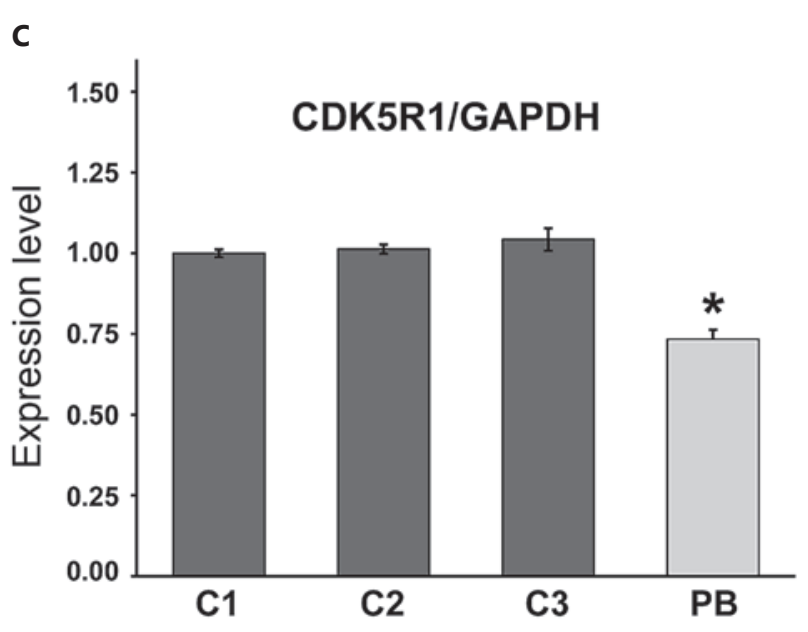

(build 37/hg19: February 2009). The size of the deletion is $196 \mathrm{~kb}$. C Relative level of CDK5R1 transcript in leukocytes of the proband ( $\mathrm{PB}$, light gray) and 3 unaffected controls matched by sex and age (C1-C3, dark gray). The expected $\Delta \Delta \mathrm{Ct}$ ratio is $0.5,1$, and 1.5 when one, two or three copies are expressed, respectively. Each point represents the mean \pm SD of 3 different determinations. Statistical significance versus control (C1): ${ }^{*} p<0.01$.

(d2-R test and Rey complex figure). All executive functions were found in the normal range (Trail making test, Tower of London, Wisconsin card sorting test, Elithorn perceptual maze test) with the exception of phonological fluency which appears poor. Regarding the memory domain, a specific impairment in short-term verbal memory was recorded, while verbal episodic memory and word list recall were all normal (Rey auditory verbal learning task). 
In addition, long-term visual memory was normal (Rey complex figure and Benton visual retention test). No deficits were found for arithmetic skills or in mental calculus. The patient was clinically diagnosed with a high-functioning ASD due to lack of social and emotional reciprocity, poor social abilities, impairment in spontaneous nonverbal communication, deficits in eye contact, poor facial expression, and reduced mimicry [confirmed by Autism Diagnostic Observation Schedule (ADOS-2) testing].

\section{Materials and Methods}

Peripheral blood was drawn from the patient and his parents into EDTA-containing tubes. Genomic DNA was extracted using the QIAamp DNA blood mini kit (Qiagen, Valentia, CA, USA), and array CGH was performed using the SurePrint G3 Human CGH 4x180K Microarray kit (Agilent Technologies, Santa Clara, CA, USA). The deletion was confirmed by quantitative real-time PCR (qPCR) using Sybr Green methodology targeting an intronic region of the CDK5R1 gene and normalized with RNase $\mathrm{P}$ gene. Real time experiments were performed using the 7900HT Fast System (Applied Biosystems, Foster City, CA, USA). RNA was extracted from blood drawn into Tempus tubes, using the TempusTM Spin RNA isolation reagent kit (P/N 4378926, Applied Biosystems) and retrotranscribed by the High Capacity RNA-to-cDNA kit (P/A 4387406, Applied Biosystems). RNA was extracted from peripheral blood mononuclear cells (PBMCs), retrotranscribed into cDNA and used to quantify CDK5R1 mRNA levels by qPCR using Sybr Green methodology. The amount of mRNA was calculated using the $2^{-\triangle \Delta C t}$ method and expression values were normalized to the internal control gene GAPDH. The calibrator sample included mRNA from 3 healthy human controls matched by sex and age. Primer sequences are available on request. Statistical comparisons were made using unpaired Student $t$ test. $p$ values of 0.01 or less were considered to represent a statistically significant difference.

\section{Results}

A 17q11.2 de novo deletion spanning approximately $196 \mathrm{~kb}$ was detected from oligomer A_16_P03233537 (hg19, 30,750,126 bp, first deleted) to oligomer A_16_ P40794889 (30,946,150 bp, last deleted) (Fig. 1A, B). It involves the whole PSMD11 (OMIM 604449) and CDK5R1 (OMIM 603460) genes as well as the last 2 exons of the MYO1D gene (OMIM 606539) (Fig. 1A, B). The PSMD11 gene encodes for a subunit of the PA700 complex, which belongs to the $26 \mathrm{~S}$ proteasome involved in the degradation of cellular-ubiquitinated proteins [Vilchez et al., 2012]. The MYO1D gene encodes for an unconventional myosin involved in left-right patterning of visceral organs [Nagase et al., 1998]. The CDK5R1 gene is specifically expressed in the central nervous system where it activates the cyclin-dependent kinase 5 encoded by the
CDK5 gene (OMIM 123831). Consistent with the loss of one allele, a decrease in CDK5R1 mRNA level was detected by RT-qPCR in the proband's PBMCs (Fig. 1C) when compared to typically developing male control subjects of similar age. DECIPHER (http://decipher.sanger.ac.uk/) reports 10 patients with chromosome 17q11.2 deletions partially or fully overlapping the one detected in our proband. Only 3 of them $(267258,289944$, and 317985) carry microdeletions limited to $17 q 11.2$, though larger than the one detected in our proband as they range in size from 0.97 to $1.18 \mathrm{Mb}$ (Fig. 1A). These 3 patients all display intellectual disability, hypotonia, and fatigue.

\section{Discussion}

The chromosome 17q11.2 microdeletion detected in our patient falls within the larger interval associated with the NF1 microdeletion syndrome (OMIM 613675), but does not encompass the NF1 gene, which is considered the main causative gene for the syndrome [Riva et al., 2000]. The 17q11.2 deletion syndrome is characterized by an excessive number of early-onset neurofibromas, intellectual disability, cardiovascular malformations, and variable dysmorphisms [Venturin et al., 2004], all absent in our proband. The CNV detected in our patient is a rare de novo deletion not listed in the Database of Genomic Variants among CNVs found in phenotypically normal individuals. It is relatively small in size, spanning only 196 $\mathrm{kb}$, and no patient carrying similarly sized CNVs is listed in the DECIPHER database. This microdeletion encompasses 3 RefSeq genes, namely PSMD11, CDK5R1, and the last 2 exons of MYO1D. These genes are not currently present in the Simons Foundation website. PSMD11 and MYO1D are ubiquitously expressed, whereas CDK5R1 is highly expressed in the cerebral cortex and to a lesser extent in the mucosa of the esophagus, in the small intestine, the spleen and in PBMCs [GTEx Consortium, 2013]. Among these 3 genes, CDK5R1 has a specific role in neurodevelopment and in higher brain functions thus appearing as the most interesting candidate to explain the patient's phenotype. The CDK5R1 gene is listed in another important bioinformatic autism web-based resource called "AutismKB" (http://autismkb.cbi.pku.edu. cn/index.php), which lists the CDK5R1 gene as a candidate gene for ASD. Evidences for its role in ASD include (1) one microarray gene expression study in children with ASD [Gregg et al., 2008] and (2) six linkage studies of which the most significant is by Sutcliffe et al. [2005]. The first study reports a dysregulation of the CDK5R1 gene
Lintas et al. 
expression in autistic children compared to age- and sexmatched typically developed controls, whereas linkage studies demonstrated a strong association of the $17 \mathrm{q} 11.2$ region with ASD.

The protein encoded by $C D K 5 R 1$, known also as p 35 , is necessary for the activation of $C D K 5$, the cyclin-dependent kinase 5, in the brain. The CDK5/CDK5R1 complex is required for neurite outgrowth, cortical lamination, and dendritic spine morphogenesis. $C d k 5 r$ null mice display severe cortical lamination defects and suffer from seizures and adult mortality [Chae et al., 1997]. Abnormal neocortical development characterized by inverted layering and branched migration instead of classical linear radial migration was also reported in C $d k 5 r$ null mice [Gupta et al., 2003]. In addition to neurodevelopmental defects, null mice showed motor coordination deficits and impaired long-term synaptic plasticity [He et al., 2014]. Consistent with low expression of CDK5 and CDK5R1, delayed responses to painful thermal stimulation in primary afferent nociceptive $\mathrm{C}$ fibers in the knockout mouse dorsal root ganglia were also reported [Pareek et al., 2006]. The CreER-Cdk5r1 conditional knockout mice have defective spatial learning and memory along with decreased anxiety-like behavior [Mishiba et al., 2014]. The mouse phenotype results from a reduction in the spine density of pyramidal neurons, reduced sensitivity to synaptic input, and defective long-term depression induction in the hippocampus. In the light of this evidence, the $25 \%$ decrease in CDK5R1 mRNA detected in the PBMCs of our patient, if paralleled by similar reductions in the central nervous system, may explain his peculiar neuropsychological profile characterized by moderate deficits in communication and social interaction, reduced pain sensitivity, and motor clumsiness. The CDK5R1 gene has been proposed as a candidate gene for intellectual disability in the 17q11.2 deletion syndrome [Venturin et al., 2004]. This hypothesis finds support in a recent report by Osio et al. [2018] describing the 3 abovementioned unrelated patients listed in the DECIPHER database, affected by intellectual disability, hypotonia, and fatigue, who carry overlapping de novo $17 \mathrm{q} 11.2$ microdeletions (Fig. 1A). The smallest region of overlap between these 3 patients contains 7 genes including CDK5R1, which is, considered by the authors, a good candidate for explaining the developmental delay. In mutation screenings involving patients with idiopathic intellectual disability, several variants in the $3^{\prime}$-UTR and in the coding region of the CDK5R1 gene were found [Venturin et al., 2006; Moncini et al., 2016]. However, the functional significance of some of these mutations is controver-

CDK5R1 Deletion in a High-Functioning Autistic Patient sial. Some studies report altered gene expression levels and/or altered protein function [Moncini et al., 2016], whereas others found no significant differences in the biochemical and neurodevelopmental properties of the mutant and wild-type forms of CDK5R1 [Takada et al., 2015].

In summary, our data suggest that the heterozygous deletion of the CDK5R1 gene yields a decrease in CDK5R1 mRNA level in our proband, compatible with phenotypic traits less severe than previously described intellectual disabilities. Our patient in fact displays high-functioning autism, short-term verbal memory deficits, difficulties in motor coordination, and pain hyposensitivity. Further research will have to address whether similar heterozygous deletions reduce $C D K 5 R 1$ gene expression to a similar or to a different extent in different patients, possibly supporting an epigenetic modulation at this locus as primarily responsible for clinical variability in penetrance. Alternatively, deletion size (i.e., other deleted loci) or epistatic interactions with genetic variants located in other chromosomes may contribute to interindividual differences in phenotypic severity.

\section{Acknowledgment}

We thank the patient and his family for their cooperation and Marta Tiberi for technical assistance with qPCR. This work was supported by the Italian Ministry of Health (NET-2013-02355263).

\section{Statement of Ethics}

Informed consent for the present study and image publication was obtained from the patient. The authors have no ethical conflicts to disclose.

\section{Disclosure Statement}

The authors declare no conflicts of interest.

American Psychiatric Association: Diagnostic
and Statistical Manual of Mental Disorders,
ed 5 (American Psychiatric Publishing, Ar-
lington 2013).
Chae T, Kwon YT, Bronson R, Dikkes P, Li E, Tsai
LH: Mice lacking p35, a neuronal specific ac-
tivator of Cdk5, display cortical lamination
defects, seizures, and adult lethality. Neuron
18:29-42 (1997).
Gregg JP, Lit L, Baron CA, Hertz-Picciotto I, Walk-
er W, et al: Gene expression changes in children
with autism. Genomics 91:22-9 (2008).


GTEx Consortium: The Genotype-Tissue Expression (GTEx) project. Nat Genet 45:580-585 (2013).

- Gupta A, Sanada K, Miyamoto DT, Rovelstad S, Nadarajah B, et al: Layering defect in p35 deficiency is linked to improper neuronal-glial interaction in radial migration. Nat Neurosci 6:1284-1291 (2003).

- He X, Ishizeki M, Mita N, Wada S, Araki Y, et al: $\mathrm{Cdk} 5 / \mathrm{p} 35$ is required for motor coordination and cerebellar plasticity. J Neurochem 131: 53-64 (2014).

Mishiba T, Tanaka M, Mita N, He X, Sasamoto K, et al: Cdk5/p35 functions as a crucial regulator of spatial learning and memory. Mol Brain 7:82 (2014).

Moncini S, Castronovo P, Murgia A, Russo S, Bedeschi MF, et al: Functional characterization of CDK5 and CDK5R 1 mutations identified in patients with non-syndromic intellectual disability. J Hum Genet 61:283-293 (2016).

- Nagase T, Ishikawa K, Suyama M, Kikuno R, Miyajima N, et al: Prediction of the coding se- quences of unidentified human genes. XI. The complete sequences of 100 new cDNA clones from brain which code for large proteins in vitro. DNA Res 5:277-286 (1998).

- Osio D, Rankin J, Koillinen H, Reynolds A, Van Esch H: Interstitial microdeletion of $17 \mathrm{q} 11.2$ is associated with hypotonia, fatigue, intellectual disability, and a subtle facial phenotype in three unrelated patients. Am J Med Genet A 176:209-213 (2018).

- Pareek TK, Keller J, Kesavapany S, Pant HC, Iadarola MJ, et al: Cyclin-dependent kinase 5 activity regulates pain signaling. Proc Natl Acad Sci USA 103:791-796 (2006).

Persico AM, Napolioni V: Autism genetics. Behav Brain Res 251:95-112 (2013).

-Riva P, Corrado L, Natacci F, Castorina P, Wu BL, et al: NF1 microdeletion syndrome: refined FISH characterization of sporadic and familial deletions with locus-specific probes. Am J Hum Genet 66:100-109 (2000).

- Sutcliffe JS, Delahanty RJ, Prasad HC, McCauley JL, Han Q, et al: Allelic heterogeneity at the sero- tonin transporter locus (SLC6A4) confers susceptibility to autism and rigid-compulsive behaviors. Am J Hum Genet 77:265-279 (2005).

- Takada S, Mizuno K, Saito T, Asada A, Giese KP, Hisanaga S: Effects of p35 mutations associated with mental retardation on the cellular function of 35-CDK5.PLoSOne 10:e0140821 (2015).

Venturin M, Guarnieri P, Natacci F, Stabile M, Tenconi R, et al: Mental retardation and cardiovascular malformations in NF1 microdeleted patients point to candidate genes in 17q11.2. J Med Genet 41:35-41 (2004).

-Venturin M, Moncini S, Villa V, Russo S, Bonati MT, et al: Mutations and novel polymorphisms in coding regions and UTRs of CDK5R1 and OMG genes in patients with non-syndromic mental retardation. Neurogenetics 7:59-66 (2006).

Vilchez D, Boyer L, Morantte I, Lutz M, Merkwirth $\mathrm{C}$, et al: Increased proteasome activity in human embryonic stem cells is regulated by PSMD11. Nature 489:304-308 (2012). 